\title{
A Development of Agility Mode in Cardiopulmonary Resuscitation Learning Support System Visualized by Augmented Reality
}

\author{
Keisuke Fukagawa*, Yuima Kanamori, Akinori Minaduki \\ Medical Informatics Laboratory, Kushiro Public University, 085-8585, Japan
}

\author{
A R T I C L E I N F O \\ Article history: \\ Received: 16 August, 2018 \\ Accepted: 17 September, 2018 \\ Online: 14 November, 2018
}

Keywords :

Cardiopulmonary Resuscitation

Visualization

Augmented Reality

\begin{abstract}
A B S T R A C T
This paper showed visualization of technics about cardiopulmonary resuscitation contributed to acquiring the skills and understanding them. Especially, this system is focused on the individuality in each object (men, women, and babies) There are problems of a general cardiopulmonary resuscitation (CPR) training because learners are generally taught by instruction based on a subjective judgment. Because of this way, they are difficult to get a knack about the CPR themselves.

Our system solved these problems by using Kinect (Augmented Reality) and Wii Balance Board (Weight Scale) when it calculates pressure and posture. They can understand the CPR training as fixed information. The system also expresses the features of compression as results whether the posture is extension or bending. However, it cannot evaluate a process of the compression. In the inspection, 84 people wrote a questionnaire which focused on impression of before and after. This questionnaire expressed two things. The one is that general public don't know the presence of CPR for infants. The other is that visualization was effective and enjoyable. As future works, Another function is going to be added to the present system to evaluate CPR in a process.
\end{abstract}

\section{Introduction}

Cardiopulmonary resuscitation (hereinafter referred to CPR), which maintains opportunities for life support by performing chest compressions on victims who are in a state of cardiopulmonary arrest, is a technique of life saving performed before and after using AED. This technique is well known. For example, some instructors teach the skills to learner in a general CPR training. In this case, learners can understand CPR. However, that tends to be subjective because the instructors teach them without using objective indicators. Because of this way, there are some problems in which learners push mannequins excessively. In addition, there are regulations about CPR in each object (men, women, infants, older people) but most people who participate the training don't learn them.

This new system is a visualized learning application of CPR which can calculate the pressure of compression and the angle of elbows with Augmented Reality (hereinafter referred to AR). It is possible to evaluate CPR in 1 minute. Learners of this system can

*Keisuke Fukagawa, Public University, 4-1-1 Ashino Kushiro City Hokkaido, Japan, +81-80-1275-1936, keiry.happylife2525@gmail.com also get the objective feedback after the training. The system is also constructed another function (it is called agility mode). By this function, learners can understand individuality of CPR in each object.

In a research about this paper with experienced or clarified nursery teachers and medical personnel, the questionnaire verified the usefulness of this system and concentrated on two things. The first one is how much ratio of general public know the presence of CPR for infants, the other one is whether the design of this system is useful or not.

\section{Related Researches}

There are regulations and previous researches on CPR. It should be done as soon as possible by proper pressure of compression and tempo. Especially, the criterion on pressure of compression is different in each object (men, women, infants, older people) Our research is also based on the criteria in previous ones of World Health Organization (hereinafter referred to WHO), American Heart Association (hereinafter referred to AHA), and the Japan Resuscitation Council and the Japan Foundation for Emergency Medicine. 


\subsection{The Situation of CPR in Japan}

Types of commonly performed CPR in Japan are Basic Life Support (BLS)/Advanced Cardiovascular Life Support (ACLS) by AHA and BLS/ALS (Advanced Life Support) by the Japan Resuscitation Council and the Japan Foundation for Emergency Medicine. According to the Guideline Update 2015[1], as the situation clearly differs between in-hospital cardiac arrest (IHCA) and out-of-hospital cardiac arrest (OHCA), patients are divided into OHCA and IHCA cases and appropriate treatment methods are specified for both. This paper focuses on increasing the quality of lay rescuers $\mathrm{CPR}$ and describes a system development for OHCA.

\subsection{The probability of Survival}

The CPR is an efficient way to supply oxygen to brains when someone is in a cardiac arrest. If a person who in this situation, it will occur permanent damage for him/her. Dr. Drinker made the named Drinker curve on the influence in 1966 and was reported by WHO about one. According to previous researches, the positive effect of CPR is stronger if it is started in the cardiac arrest as soon as possible. The probability of survival also increases compared with nothing as times go [Fig.1].

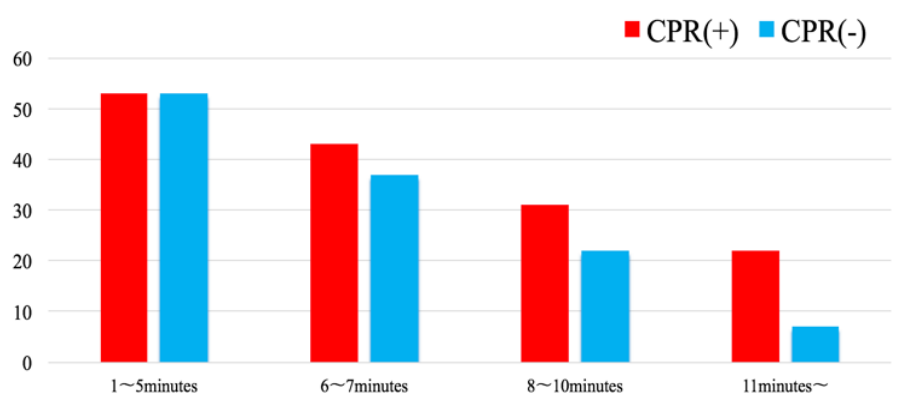

Figure. 1 This graph shows the effect of CPR in each phase. The vertical axis means the probability of survival and the horizontal axis does elapsed time after a cardiac arrest. Also, $\mathrm{CPR}(+)$ means a case of this resuscitation done, and $\mathrm{CPR}(-)$ does that CPR was not done.

\subsection{Regulations of $C P R$}

In the CPR, learners have to acquire skills and obey regulations to do it properly. First of all, the compression depth is at least $5 \mathrm{~cm}$ but no more than $6 \mathrm{~cm}$. Secondly, learners should perform at 100-120 repetitions per a minute [2], [3]. Decompression isn't done properly if the number of the compression is more than 120 .

The decompression plays an important part to do the CPR. Third, they do the CPR in an unceasing performance with minimal interruption time within 10 seconds. This is because the probability of survival de-creases. Forth, they have to do it with being perpendicular from the elbow jointing the base of the palm to do the CPR in a proper pressure. Fifth, the pressure of compression is between $40 \mathrm{Kg}$ and $50 \mathrm{Kg}$ (It is for men.) in order not to break breastbones. In general CPR, breaking breastbones aren't bad to save a life. However, this breaking make patients take a long period to recover.

\subsection{The Individuality of $C P R$ for Infants}

In the JRC resuscitation guideline summarized by the Japan Resuscitation Council, the two-fingers compression method is recommended as a CPR method for infants. The method is to compress by two fingers in the middle of the chest and press the sternum with one hand [4]. CPR to adults is also different from using only one hand. In consideration of this difference, the system is constructed so that the KINECT sensor can detect only one arm of the learner. Regarding pressure criteria for infants, the pressure is measured when the same portion of a mannequin similar to the actual infant's rib cage anterior-posterior diameter strength developed by Laerdal pressed to sink one-third of the whole. Besides, the value measured by this was set as the pressure detection standard of this system.

\section{System Outline}

This system is developed by 6 components and Microsoft Visual Studio 2017 and .NET Framework 4.5 with the C\# language. There are a Kinect for windows version2 (1), a Wii Balance Board (2), a Communication Monitor (HDD) (3), a Mini Anne (CPR/ AED learning tool kit) (4), a Bluetooth USB adaptor (5), and Control PC (Windows OS) (6) in our system[Fig.2].

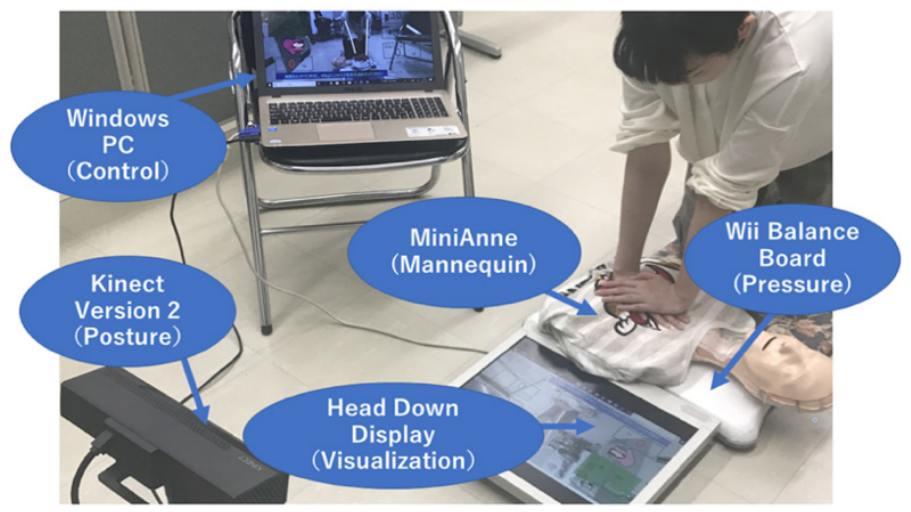

Figure 2 This figure shows the arrangement of our system. Learners can objectively understand the pressure of compression and the posture. They can do the CPR training with seeing the HDD.

\subsection{Data Flow}

Learners can experience a real CPR training themselves with using our system. The data flow is four steps [Fig.3]. The first one is choosing a target in men, women, babies (They have to choose right or left because the CPR for babies are done by only one hand).

Next, the system sets the level of sensitivity on the pressure of compression. The criteria on pressure compression and shape of men, women, and babies are different, so the system should change in each object. As third step, the AR of Kinect reads their body, and learners can start doing the CPR training. Lastly, the system counts whether their compression is extending or bent position pressure. Each compression of learners is counted. 


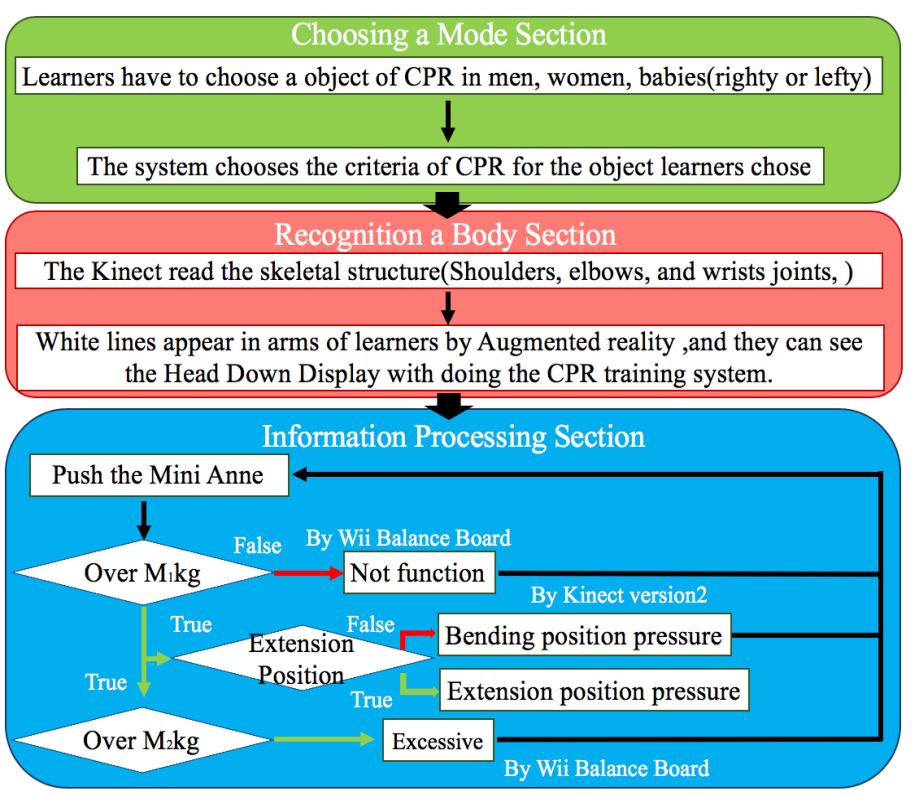

Figure 3 This chart shows the outline of our system. In the third section, Mı means a minimum and $\mathrm{M}_{2}$ does a maximum. In case of the CPR for infants, the criteria of minimum are $5 \mathrm{~kg}$, and the one of maximum is $10 \mathrm{~kg}$. This regulation is based on a research of Laerdal.

\subsection{Feedback System}

After finishing the training, our system reports their compression features. The results are classified by frequency of learner's compression and posture into five types. The posture of compression are counted in extension or bending position pressure [Fig.4].

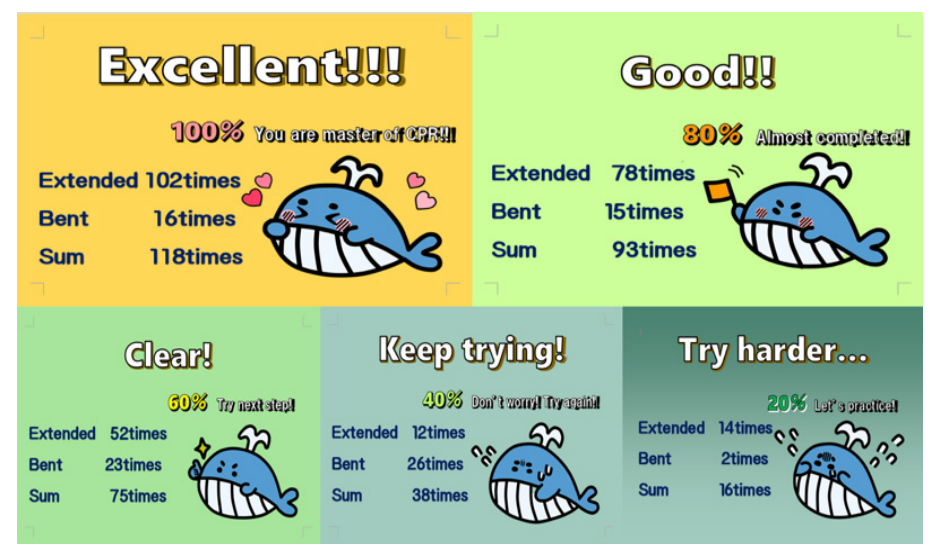

Figure 4 The results are determined by whether the counts of extension position pressure are enough or not.

\subsection{Motion Capture}

This system can calculate whether learner's posture is extension or bending by AR. Kinect reads 26 joints in human body in initial setting, but it is controlled [Fig.5, Fig.6]. Learners can see the angles from shoulders to elbows and elbows to wrists in 6 points. Also, learners should obey regulations which was found in a process of the demonstrations below to use the system properly.

1. Tie your hair if you have long one.

2. Don't use in front of white walls.
3. Don't use the system outside.

4. Start to use the system from the pose of T.

5. Make your elbows straight (in an extension position).

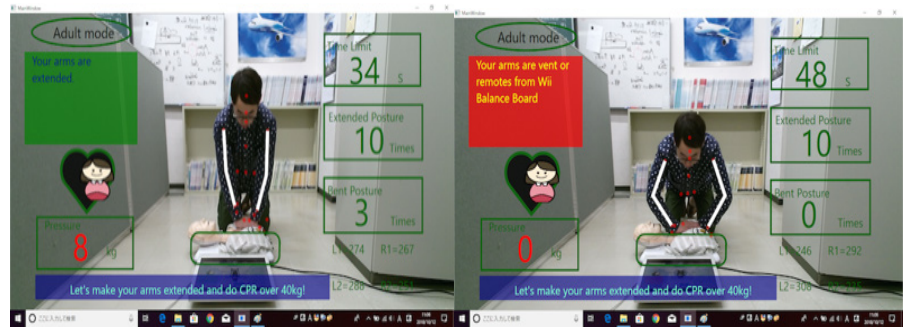

Figure 5 This left picture shows the situation of extension position pressure. This heart mark is also usually black. However, the heart mark becomes pink if the learners do compression over criteria. This right one shows the situation in the bending position pressure. If so, it is hard to press the Mini Anne in an appropriate compression force. This is because the bending position pressure needs power to do one properly more than the extended position one.

\subsection{Agility Mode(Individuality)}

An agility mode function was developed so that training suitable for the individuality in each object can be selected by expanding the system by the target age groups and gender classification. As a result, the learner can select CPR targets to be learned on the home screen [Fig. 6]. The subjects are classified into, men, women, and infants, and are displayed on the home screen accordingly. In particular, when choosing CPR training for infants, considering the difference in domestic hand for each learner, the interface was arranged whether it is for right-handed or left-handed, with classification about the individuality of each target.

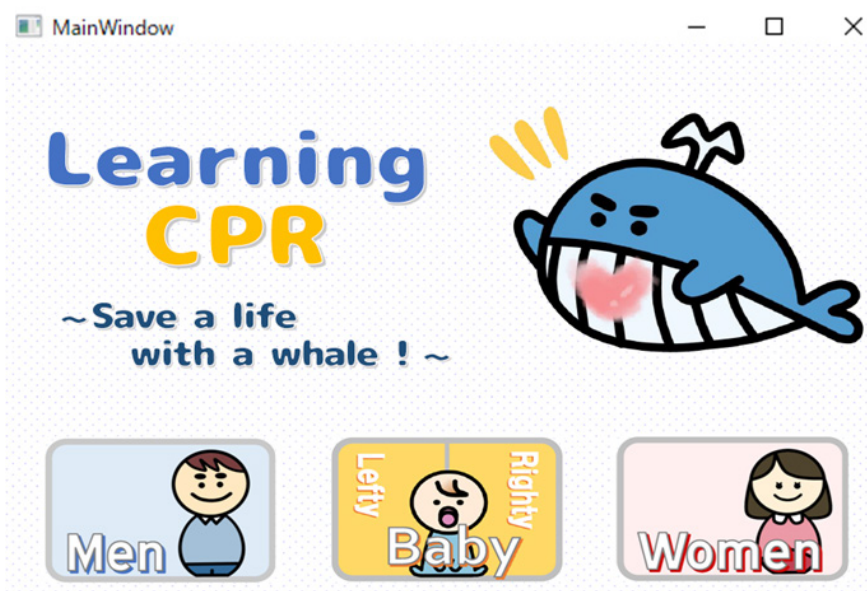

Figure 6 This shows the start screen of our system. Learners can select the object they want to experience.

\section{Results}

In this verification, CPR training was experienced with 84 people (33men, 51women) and they answered closed questions. The questionnaire was mainly surveyed whether the people know the CPR for infants or not and the effect of visualization, and the impression of the CPR training before and after (Table.1). 


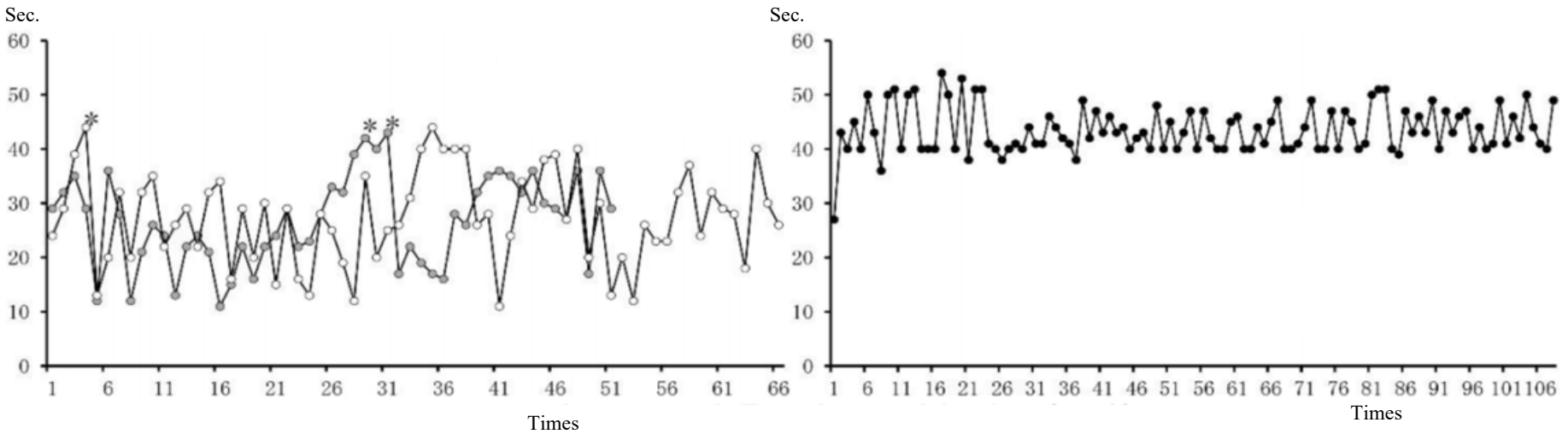

Figure 7 This graph (the object was men in this case) is that there is difference between before the CPR training (the left one) and after one (the right one) by one research subject. The vertical axis is a pressure of compression $(\mathrm{kg})$ and, the horizonal axis means the number of compression(times). The white line is the first, and the gray one is the second. Also, the black one is the third. This verification clearly represents an effect by using the CPR training system because the pressure of compression of the research subject became more stable in an appropriate level, and the number of compression increased. In addition, this asterisk is the case of bent position pressure.

Table.1 The questionnaire of our system

\begin{tabular}{|l|l|}
\hline & Question Items \\
\hline Q1 & Have you ever participated in the lecture class of CPR training? \\
\hline Q2 & $\begin{array}{l}\text { Did you know a specific way of CPR for infants before participating } \\
\text { the lecture? } \\
\text { (For Childcare Worker, General Public) }\end{array}$ \\
\hline Q3 & Do you mind doing the CPR for the opposite sex? \\
\hline Q4 & Was the CPR training System useful? \\
\hline Q5 & Were you able to study the CPR training with enjoying? \\
\hline
\end{tabular}

\section{Discussion}

According to this survey[Table.2], this system contributed two things. First of all, it showed that about $88 \%$ of general public didn't know the CPR for infants even if $75 \%$ of them have participated in the lecture. Second, about $92 \%$ of respondents could feel enjoyable and think it was useful. The system was actually focused on the design by using a whale because we wanted everyone to study with enjoying. However, the number of people with whom we trained the CPR was not enough to prevail it about infants and women.

Table 2 The Results of the questionnaire

\begin{tabular}{|l|l|l|}
\hline Question & YES & NO \\
\hline Q1 & $75.00 \%$ & $25.00 \%$ \\
\hline Q2(All) & $45.31 \%$ & $54.69 \%$ \\
\hline Q2(Only General Public) & $12.12 \%$ & $87.88 \%$ \\
\hline Q2(Only Childcare Worker) & $93.10 \%$ & $6.900 \%$ \\
\hline Q3(Men) & $52.17 \%$ & $47.83 \%$ \\
\hline Q3(Women) & $19.51 \%$ & $80.49 \%$ \\
\hline Q4 & $98.44 \%$ & $1.56 \%$ \\
\hline Q5 & $92.19 \%$ & $7.81 \%$ \\
\hline
\end{tabular}

\section{Conclusion}

In this paper, objects (men, women, babies) of this system could be classified as different ones. This progress is a first step to prevail the presence of CPR for general public. Another function is going to be added to the present system to evaluate $\mathrm{CPR}$ in a process.

\section{Conflict of Interest}

The authors declare no conflict of interest.

\section{Acknowledgment}

This research was started by Medical Informatics Laboratory of Kushiro Public University in 2009. As a verification, Kushiro Kojinkai Nursing School, Ashino Nursery School, Kushiro City Childcare Support Center Eastern Branch helped us. We appreciate these organization.

\section{References}

[1] Japan Resuscitation Association, "JRC Resuscitation Guidelines 2015 Online Version, Chapter 2 Adult Secondary Life Support Measures (ALS)" (2015)

[2] Kralj E, Podbregar M, Kejžar N, Balažic J., "Frequency and number of resuscitation related rib and sternum fractures are higher than generally considered.Resuscitation".2015;93:136141.doi:10.1016/j.resuscitation.201 5.02 .034

[3] A.E.Tomlinson, J.Nysaether, J.KramerJohansen,P.A.Steen,E.Dorph, "Compression force-depth relationship during out-of-hospital cardiopulmonary resuscitation", doi: 10.1016/j.resuscitation.2006.07.017

[4] Japan Resuscitation Association, "JRC Resuscitation Guidelines 2015

[5] Online Version, Chapter 3 Child Resuscitation (PLS)" 